Hamid Mokaddem, Ce souffle venu des Ancêtres...

L'oeuvre politique de Jean-Marie Tjibaou (1936-1989)

Expression - Province Nord, Nouméa, 2005

\title{
Adriano Favole
}

\section{(2) OpenEdition}

Édition électronique

URL : http://journals.openedition.org/jso/673

DOI : $10.4000 /$ jso.673

ISSN : $1760-7256$

\section{Éditeur}

Société des océanistes

Édition imprimée

Date de publication : 1 décembre 2006

Pagination : 221-222

ISSN : 0300-953x

\section{Référence électronique}

Adriano Favole, «Hamid Mokaddem, Ce souffle venu des Ancêtres... L'oeuvre politique de Jean-Marie Tjibaou (1936-1989) », Journal de la Société des Océanistes [En ligne], 122-123 | Année 2006, mis en ligne le 23 avril 2008, consulté le 24 septembre 2020. URL : http://journals.openedition.org/jso/673 ; DOI : https://doi.org/10.4000/jso.673 
nelle » et les dévoilements inattendus, le vécu à la limite du dicible, les données ethnographiques et l'expérience rapportées vers l'autre côté de la planète et leur parfois difficile mise en place dans les débats anciens et les modes, jeux de pouvoir et attentes du moment.

\section{BIBLIOGRAPHIE}

BonNEMÈre Pascale, 1996. Le pandanus rouge: Corps, différence des sexes et parenté chez les Ankave-Anga (Papouasie Nouvelle-Guinée), Paris, CNRs/Éditions de la maison des sciences de l'homme.

BonNEMÈre Pascale (ed.), 2004. Women as Unseen Characters. Male Ritual in Papua New Guinea, Philadelphie, University of Pennsylvania Press.

Denis Monnerie, université Marc Bloch, Strasbourg

Hamid MoKaddem, 2005, Ce souffle venu des Ancêtres... L'xuvre politique de Jean-Marie Tjibaou (1936-1989), Nouméa, Expression - Province Nord.

L'ouvrage d'Hamid Mokaddem se révèle être une contribution originale et novatrice à la figure de Jean-Marie Tjibaou. Si, comme le mentionne l'auteur dans sa préface («L'ordre du discours »), l'écriture est « risque » et « fiction" - dans le sens de « construction » d'un parcours non prévisible -, le texte réussit, pour sa part, brillamment l'examen. Mokaddem, en impliquant le lecteur dans un discours vigoureux, établit un lien entre la «courbe existentielle» (p. 17) du personnage Tjibaou et la «trajectoire» historique à laquelle il dut se rapporter. Le texte n'est d'ailleurs pas organisé en chapitres de manière rigide et canonique :

«Ce livre n'est agencé ni en chapitres ni en parties, mais en lignes, lesquelles bifurquent, s'entrecroisent et parfois se plient, formant des plans. Ces plans ne sont pas statiques. $\mathrm{Ce}$ sont des retours cycliques autour d'un axe, la ligne chronologique. » (p. 13)

Le style de Mokaddem est original et riche de références savantes et interdisciplinaires : l'auteur mêle, en effet, avec aisance l'ethnologie, la sociologie et la philosophie. Ses innovations stylistiques sont souvent agréables et surprenantes, alors que certains de ses choix apparaissent, quant à eux, discutables. L'absence d'un index général suscite par exemple une interrogation, tandis que la subdivision de la bibliographie en genres («Anthropologie», « Droit», " Économie », " Histoire », etc.) met en difficulté le lecteur non spécialiste.

Le volume se fonde sur des sources originales qui permettent d'enrichir considérablement notre connaissance de la biographie de Jean-Marie Tjibaou. Il s'agit non seulement d'environ trente interviews accordées par des personnages - parmi lesquels des hommes politiques, des chercheurs et des membres de la famille - qui ont fréquenté et collaboré avec le leader kanak, mais aussi d'archives peu explorées ou restées inédites à ce jour, accompagnées de recherches de terrain effectuées personnellement par l'auteur. Certains discours inédits de Tjibaou sont intégralement publiés dans la partie finale du livre (« Transcriptions d'inédits de dits, paroles et propos de Jean-Marie Tjibaou, 1978-1989») et la «génialité » de Tjibaou (« génie au sens que lui ont conféré Kant et Hegel, à savoir une singularité propre à un peuple ayant force d'universalisation et la capacité d'inventer des nouvelles règles ", p. 21) transparaît dans toute sa force au travers des neuf lignes qui composent le texte. La première ligne ( $\mathrm{La}$ reformulation permanente kanak») retrace la genèse et développe l'un des concepts les plus célèbres et chers à Tjibaou, à savoir celui de la « reformulation permanente ». Mokaddem précise que cette notion a tout d'abord une signification biographique puisque Tjibaou fut en effet séminariste, prêtre, étudiant en sociologie et ethnologie, animateur et enfin homme politique. La reformulation permanente, avant même de devenir un projet politique, est la caractéristique de la personne humaine considérée par Tjibaou comme un faisceau de possibilités et de relations. La reformulation permanente est aussi la capacité de ne pas se faire emprisonner dans le passé tout en évitant la fuite vers la modernité comme l'attestent les propos mêmes de Tjibaou :

« Notre but est d'affirmer les richesses de nos propres modèles et de laisser grand ouvert pour nous, l'éventail des choix culturels permettant aux gens de construire une personnalité. » (p. 36)

Tjibaou, comme tout être humain, ne naît pas dans un « vide ", mais bien dans un lieu et à une époque marquée par l'histoire (Ligne II, « Une ligne invariable »). Quelle influence eurent donc les vicissitudes historiques de la lignée Cibau sur Jean-Marie telles que le meurtre de sa grand-mère lors des événements de 1917, les pérégrinations successives du «nom» Cibau dans différentes zones de la Grande Terre et le meurtre de deux de ses frères au cours de l'embuscade de 1984 ? Sans oublier l'influence de la mission mariste et notamment du prêtre Alphonse Rouel qui confia au père de Tjibaou la responsabilité de la première école de Tiendanite et qui envoya Jean-Marie au petit séminaire de Canala (1945), lui ouvrant ainsi la voie de la prêtrise ? Mokaddem formule une hypothèse originale : l'adhérence de Tjibaou à l'Église doit être interprétée suivant l'optique d'une politique des alliances qui lia pendant une certaine période la famille Tjibaou à la mission mariste par l'intermédiaire de Rouel. Jean-Marie se tournera vers l'état laïque de manière significative seulement suite à la mort de Rouel qui caractérise la fin de l'alliance.

L'histoire conditionne lourdement la conduite des hommes, mais la grandeur des personnages illustres réside précisément dans leur capacité à orienter l'histoire (Ligne III, « Brisures »). La vie de Tjibaou est, en effet, riche de ruptures. Sa participation au petit séminaire de Canala lui offre la possibilité d'étudier tout en l'éloignant en même temps de sa famille et de son village natal pendant une dizaine d'années. À son retour en 1956 à Tiendanite, il se sent « étranger » au sein de sa propre société en raison de la perte de la maîtrise de sa langue maternelle. Une fois encore, 
Mokaddem réussit à exprimer, à partir de cette donnée biographique, une réflexion de caractère plus général sur l'expérience du colonialisme, sur la « schizophrénie » du colonisé qui devient étranger de sa propre culture, comme en témoignent des auteurs tels que Franz Fanon et Aimé Césaire. Dans cette partie centrale digne d'intérêt, le volume suit de près la ligne chronologique : après Canala et différentes expériences en tant que novice vécues à l'Ile des Pins, à Thio et à Lifou, Tjibaou rejoint le séminaire de Paita. La vie de séminariste se compose d'étude rigoureuse, de contrôle du corps et des émotions et d'isolement du monde extérieur. Paradoxalement et contre les intentions mêmes des autorités religieuses, le séminaire deviendra également un lieu de rencontre entre les futurs protagonistes de la lutte indépendantiste (Eloi Machoro, Bernard Lepeu). Son départ du séminaire et son entrée en tant qu'aumônier militaire et deuxième vicaire à la cathédrale de Nouméa constitueront pour Tjibaou une expérience décisive : confronté «aux rejets, exclus et marginaux du corps de la société coloniale » (p. 82), aux collusions entre monde religieux et monde politique, il en viendra à « rompre » définitivement avec l'Église catholique.

L'irruption de Tjibaou dans la vie politique se produira plus tard, au terme d'un «long tour» que Mokaddem analyse aux deux lignes suivantes (Ligne IV, «Se former, s'exiler» et Ligne V, « De l'idéal en politique »). La formation de Tjibaou à Lyon et à Paris, ses rapports avec l'ethnologie (Roger Bastide, Jean Guiart), la sociologie et l'économie sont traités par l'auteur sous une perspective originale, enrichie de détails inédits de la vie de Tjibaou étudiant. La découverte de Maurice Leenhardt fait l'objet d'une attention particulière : selon Mokaddem, l'influence du prêtre/ethnologue protestant sur Tjibaou a souvent été surestimée et, tandis que Tjibaou n'a de cesse de souligner l'importance de Leenhardt dans l'œuvre de valorisation des cultures kanak, il maintient toutefois une relation «distante, respectueuse » (p. 110) à son égard. Leenhardt est pour lui un point d'appui, un instrument de lutte et non un point de repère théorique (l'anthropologie appliquée et l'ethnopsychiatrie constitueront des références théoriques plus importantes). Un élément, en aucun cas secondaire, de l'œuvre de Leenhardt représentera toutefois une source d'illumination décisive pour le jeune Tjibaou : il s'agit de la catégorie de « personne » qu'il véhiculera du domaine du mythe au domaine de la politique.

Le retour de Tjibaou en Nouvelle-Calédonie est de nouveau caractérisé par un entrelacement entre biographie et histoire : son mariage avec Marie-Claude coïncide avec sa prise de conscience de l'importance des mouvements féminins dans le processus de renaissance culturelle kanak. C'est à cette époque que s'affirme le terme "Kanak» : ce sont les années de Kanaké, le héros de différents mythes qui devient la métaphore et la métonymie de la croissance de la pensée nationaliste. Tjibaou déploie son énergie dans un projet de nature plus culturelle (éthique et esthétique) que directement politique : la bataille pour la valorisation du patrimoine qui se couronnera lors de Melanesia 2000. Le projet culturel différencie Tjibaou de la gauche extrémiste qui n'hésitera pas à prendre ses distances vis-à-vis du Festival.

Aux lignes VI et VII («Maxa, l'acte révolutionnaire »; "Construire, déconstruire, reconstruire. $G i$ meen walek »), Mokaddem se fait historien. Tjibaou fait irruption dans l'histoire des Kanak, de la Nouvelle-Calédonie et du monde entier à partir de son expérience de maire de la commune d'Hienghene. Un aspect de l'interprétation réalisée par Mokaddem concernant le Tjibaou homme politique nous semble particulièrement intéressante. Il s'agit de la lecture du célèbre concept d'《interdépendance » réalisée à la lumière d'une théorie et d'une pratique « native » de la démocratie. Les interdépendances de Tjibaou ne sont pas seulement le fruit d'une conception relationnelle de l'être humain qui l'incite à refuser le repliement sur le « nous, Kanak ». L'interdépendance est une théorie et une pratique de la concertation, le refus d'une conception de la démocratie comme loi du nombre en faveur d'une théorie du débat, du dialogue entre les différences forces (à une échelle différente, de la tribu à l'État) impliquées dans le processus politique. « Il agit et œuvre en véritable politique, conciliant les structures sociales endogènes avec les modèles exogènes dominants » (p. 166).

Les deux dernières lignes du livre (VIII, « Ce souffle venu des ancêtres... »; IX « Figure et grandeur du politique en Océanie ») sont consacrées au « style » de Tjibaou. La notion de style se décline en trois niveaux : le niveau existentiel (le charisme de Tjibaou, sa capacité à dialoguer, l'intolérance envers les extrémistes), le niveau formel (l'attention accordée à la parole, l'utilisation de figures rhétoriques) et le niveau politique (la concertation, la recherche du consentement). La grandeur de Tjibaou réside précisément dans sa capacité à avoir élevé au rang de projet politique une conception de la culture comme « art de vivre », un style devenu réalité politique.

Adriano Favole, université de Turin 